\title{
Conservation Genetics and Gut Microbial Communities' Variability of the Critically Endangered European Mink Mustela Lutreola: Implications for Captive Breeding Programs
}

Pauline ML van Leeuwen ( $\nabla$ vanleeuwenpauline3@gmail.com )

Laurentian University https://orcid.org/0000-0002-9558-1359

Albrecht I. Schulte-Hostedde

Laurentian University

Christine Fournier-Chambrillon

Groupe de Recherche et d'Étude pour la Gestion de l'Environnmement

Carmen M. Aranda

Fundacion para la Investigation en Etologia y Biodiversidad

Laurie Berthomieu

Zoodyssée

Pascal Fournier

Groupe de Recherche et d'Étude pour la Gestion de l'Environnement

Johan R. Michaux

Université de Liège: Universite de Liege

\section{Research Article}

Keywords: Mustela lutreola, microbiota, MHC, immunogenetics, breeding program, genetic diversity, captivity

Posted Date: October 19th, 2021

DOI: https://doi.org/10.21203/rs.3.rs-932275/v1

License: (c) (i) This work is licensed under a Creative Commons Attribution 4.0 International License. Read Full License 
1 Title: Conservation genetics and gut microbial communities' variability of the critically

2 endangered European mink Mustela lutreola: Implications for captive breeding programs

3 Authors and affiliations: Pauline van Leeuwen ${ }^{1,2}$, Albrecht I. Schulte-Hostedde ${ }^{1}$,

4 Christine Fournier-Chambrillon ${ }^{3}$, Carmen M. Aranda ${ }^{4}$, Laurie Berthomieu ${ }^{5}$, Pascal

5 Fournier $^{3}$, Johan R. Michaux ${ }^{2}$

$6{ }^{1}$ Department of Biology, Laurentian University, Sudbury, ON, Canada;

$7 \quad{ }^{2}$ Conservation Genetics Laboratory, University of Liège, Liège, Belgium;

$8{ }^{3}$ Groupe de Recherche et d'Etude pour la Gestion de l'Environnement, Villandraut,

9 France

$10{ }^{4}$ Fundación para la Investigación en Etología y Biodiversidad, Madrid, Spain

$11{ }^{5}$ Zoodyssée, Villiers-en-Bois, France

12 Corresponding author: Pauline van Leeuwen pvan_leeuwen@laurentian.ca

13 Running title: Gut bacteria \& genetics of the European mink

14 Abstract:

15 Host's fitness can be affected by its genotype and gut microbiota, defined as the microbes

16 living in the host's intestinal tract. This study explored how the genetic diversity of the host

17 influences its bacterial communities in the context of captive breeding programs, for the

18 critically endangered European mink (Mustela lutreola). As stated by the ecosystem on a

19 leash model, loss of host genetic diversity may lead to changes in immunomodulation and

20 will therefore induce modifications of the gut microbiota. We investigated variation in the

21 gut bacteria through 16S rRNA metabarcoding, related to the genetic diversity of European

22 mink held in captivity in two breeding centers representing separate breeding stocks

23 originating from the western and eastern populations. The genetic diversity of the host was 
24 assessed through diversity analysis of the adaptive MHC class I and II genes as well as

25 neutral microsatellite markers. Results indicate lower diversity in neutral and MHC class I

26 genes for the western population, and the opposite for MHC class II. A lower MHC class

27 II gene variability led to an increase in microbial phylogenetic diversity and in abundance

28 depending on the presence of specific MHC-II motifs. Those results seem to be linked to

29 management practices that differs between the two programs, especially the number of

30 generations in captivity. Long term Ex situ conservation practices can thus modulate gut

31 microbial communities, that might potentially have consequences on the survival of

32 reintroduced animals. We suggest strategies to foster genetic diversity in captive breeding

33 program to mitigate the effects of genetic drift on those small, isolated populations.

35 Keywords: Mustela lutreola, microbiota, MHC, immunogenetics, breeding program,

36 genetic diversity, captivity

\section{$38 \quad$ Introduction}

39 More than 5,800 animal species to date are endangered, as the Earth experiences a mass

40 extinction event (Ceballos et al., 2015). Intrinsic drivers of extinction, such as genetic

41 factors, play a key role for population viability, especially when species are reduced to

42 small, isolated populations that can be negatively affected by genetic load (Hedrick, 2001).

43 In this scenario, finding a suitable mate is challenging and reproduction with related

44 individuals can occur, leading to inbreeding depression. Inbreeding has largely been

45 documented in small populations in the wild (Hedrick, 2001; reviewed in Spurgin \& Gage,

46 2019), impacting population fitness through the fixation of detrimental alleles. An increase 
47 of detrimental alleles in endangered species increases their susceptibility to extrinsic

48 ecological drivers of extinction (Frankham, 2005). One ex situ conservation tool used to

49 mitigate the decrease of genetic diversity in endangered species are Captive Breeding

50 Programs (CBPs). Captive populations of endangered species have the difficult goal of

51 ensuring the survival of stable, self-sustaining populations for later reintroduction into the

52 native habitat (Mallinson, 1995). A key challenge of CBPs is to maintain genetic diversity

53 and avoid inbreeding depression with a small number of founders (Bouman, 1977; Ralls et

54 al., 1979).

55 The majority of captive breeding schemes rely on studbooks that document pedigree

56 information within the CBPs. While studbooks can be useful to minimize inbreeding

57 effects (Pelletier et al., 2009; Witzenberger \& Hochkirch, 2011), information from

58 pedigrees can be flawed in some captive populations (Bowling et al., 2003; Marshall et al.,

59 1999; Signer et al., 1994). Molecular genetic analyses can provide more insights into the

60 relationships within captive populations and their genetic structure. Recently, genetic

61 studies of endangered species have increased, using highly variable loci non-coding for

62 fitness traits such as microsatellite markers (Witzenberger \& Hochkirch, 2011).

63 Microsatellites are known to be highly informative as a tool to measure neutral genetic

64 variation, and generally represent the extent and pattern of molecular variation within a

65 population (Selkoe \& Toonen, 2006). However, both empirical and simulated data indicate

66 that patterns of variation and divergence in adaptive traits are not always associated with

67 concomitant variation in neutral markers (Hedrick, 2001; Larson, 2012; Reed \& Frankham,

68 2001), and some conservation biologists advocate for genetic diversity analysis for

69 adaptive variation in CBPs (Hughes, 1991; Sommer, 2005). One targeted adaptive region 
70 is the Major Histocompatibility Complex (MHC) because its genes play a crucial role in

71 the adaptive immune system. Historical events such as bottlenecks and founder effects, but

72 also constraints of the mating system, such as limited sexual selection in CBPs (Schulte-

73 Hostedde and Mastromonaco 2015), can be reflected in low numbers of MHC alleles

74 (Schad et al., 2004; Hapke et al., 2004). However, in some free-ranging populations,

75 genetic variation at the MHC might persist due to balancing selection, through rare allele

76 fitness advantage, despite low levels of variability shown by neutral markers (Jarvi et al.,

77 2004; Rico et al., 2016). These studies support the difficulty of using neutral markers as

78 surrogates for variation in fitness-related loci.

79 MHC genes are considered one of the most diverse loci in jawed vertebrates and good

80 candidates for genetic diversity analysis in endangered species (Hughes, 1991). They have

81 a crucial role in adaptive immunity, by encoding proteins that bind peptide antigens and

82 present them at the cell surface to lymphocytes for their activation (Ujvari \& Belov, 2011).

83 MHC-I molecules are known to act at the intracellular level, while MHC-II molecules

84 target extracellular non-self-recognition (Ost \& Round, 2018). High genetic diversity in

85 these loci could allow targeting numerous combinations of gut microbes (Ost \& Round,

86 2018), reflected in variable immunity or tolerance among individuals through rare allele

87 and heterozygous advantage in balancing selection.

88 Within this context, Foster et al. (2017) proposed a theoretical framework known as the

89 ecosystem on a leash model, which posits that the host is under strong selection to evolve

90 mechanisms to keep the microbiota under control. The presence of a genetically diverse

91 microbiota leads to the dominance of the fastest growing microbes instead of the microbes

92 that are most beneficial to the host (Foster et al., 2017). The targeting of microbial taxa to 
93 either limit their proliferation could thus be beneficial to the host, through its adaptive

94 immune response. Bolnick et al. (2014) examined the role of MHC-II motifs (amino acid

95 sequences) in gut microbial community variation in sticklebacks (Gasterosteus aculeatus)

96 and found that common MHC motifs were linked to increases in microbial abundance and

97 diversity, and rare motifs had the opposite impacts. Similarly, the microbiota was less

98 phylogenetically diverse in individuals with high MHC-II diversity in the plumage of blue

99 petrels (Halobaena caerulea, Leclaire et al., 2018), the gut of laboratory mouse strains

100 (BALB/c, Khan et al., 2019), and the fur microbiota of fur seals (Arctocephalus gazella,

101 Grosser et al., 2019). However, no study to date has investigated the MHC-gut microbiota

102 relationships in endangered species under CBPs. We therefore hypothesize that less host

103 control, expressed by more genetically diverse gut microbes, should happen in individuals

104 with reduced genetic diversity in both neutral and adaptive markers. To test this hypothesis,

105 we investigated the genetic diversity and gut microbial community assemblages in the 106 critically endangered European mink (Mustela lutreola).

107 The European mink (E-mink) is a semi-aquatic carnivore from the Mustelidae family. Once 108 widespread throughout Europe, it was evaluated as "critically endangered" in 2011 (Maran

109 et al., 2016). There have been drastic declines in population and range, historically due to 110 overexploitation and nowadays notably driven by habitat loss, degradation and 111 fragmentation, road collisions, and the impacts of the alien American mink (Mustela vison).

112 E-mink populations are now restricted to enclaves in western France and northern Spain, 113 referred as the western population, while the eastern population is present in the delta of 114 the Danube in Romania, Ukraine and Russia (Maran et al., 2016), the latter being the focal 
115 origin of a captive breeding effort in Estonia, with successful reintroduced populations on

116 Hiiumaa Island.

117 Two major studies have documented the genetic diversity of the free-ranging E-mink

118 populations (Michaux et al., 2005; Cabria et al., 2015). The western population had a much

119 lower microsatellite genetic diversity and allelic richness compared to the eastern

120 population. The authors concluded that the western free-ranging population reached a

121 recent bottleneck, and potentially inbreeding depression due to geographic isolation.

122 However, no proof of fitness reduction in this population through inbreeding has been

123 reported as of yet (Carbonell et al., 2015). The antigen-binding site, encoded by exon 2 of

124 the DRB MHC class II gene, was also investigated in the eastern captive population by

125 Becker et al. (2009). They detected nine alleles within the 20 individuals investigated,

126 estimating low to moderate variability when comparing to other endangered species in

127 similar situations to the E-mink. However, no comparison is yet available for the captive

128 western population.

129 Both populations are currently in CBP's. The eastern breeding stock is only composed of

130 captive-born individuals for over thirty generations (Maran, pers. comm., 2021) and is

131 managed under an EAZA Ex situ Program (EEP). On the other end, the western breeding

132 stock managed by the Fundación para la Investigación en Etología y Biodiversidad (FIEB)

133 originates from the free-ranging western population captures in Spain within the last seven

134 years (i.e. seven generations), and wild-born individuals from Spain are still being 135 introduced as founders to this date. Those populations are considered as two distinct stocks 136 and are bred separately, although few cross breeds are currently being conducted. 
137 Both captive E-mink populations therefore offer a range of variation in neutral and adaptive

138 genetic diversity. Due to extreme population variation over time and the emergence of

139 small and isolated populations, the E-mink provides a unique framework to study the

140 relationship between host genetics and gut microbial communities. Following the

141 ecosystem on a leash model, the aims of this study were to (i) characterize the genetic

142 diversity in the two captive E-mink populations with neutral and adaptive genetic markers

143 as well as their gut microbial communities, (ii) examine the relationship between gut

144 microbial diversity and genetic diversity, and (iii) investigate if gut microbial community

145 structure and composition is linked to specific MHC motifs.

146 Methods

147 2.1 Sample collection and DNA extraction

148 Samples were collected from captive sexually mature mink from both populations in 2020.

149 Fourteen E-mink were sampled in the EEP conservation breeding center at Zoodyssée in

150 France (representing the eastern population), and ten E-mink were sampled in captive

151 settings at the FIEB breeding center in Spain (representing the western population) Table

152 S1]. Two mink sampled in the Spanish breeding center were wild-born individuals but

153 spent at least a year in captivity before sampling. One individual sampled in the Spanish

154 breeding center was the result of a crossbreed between western and eastern mink. For MHC

155 and microsatellite markers analysis, hair samples were collected using sterilized tweezers

156 from each animal during a routine procedure. For the gut microbiota, fresh fecal samples

157 were collected in the enclosure of each animal separately using sterilized tools and kept in

$15896 \%$ ethanol tubes at $4^{\circ} \mathrm{C}$ until further processing. As the E-mink's diet in captivity varies

159 by day, samples were collected at four occasions depending on the item fed to the animal 
160 the previous day. The diet of the E-mink from both breeding centers relied on 3 types of

161 food: trout, mice and chicken.

162 DNA from hair samples were extracted using the DNeasy Blood and Tissue Kit from

163 Qiagen using the manufacturer's protocol. DNA extractions from the fecal samples

164 collected were conducted in duplicates using the QiaAmp Mini Kit with Inhibitex (Qiagen,

165 Germany) following the manufacturer's instructions. Two blank extractions were made to

166 control for contamination during the extraction process. A mock community sample (HM-

167 783D, BEI resources) containing genomic DNA from 20 bacterial strains, at concentrations

168 ranging from 0.6 to $1400 \mathrm{pg} / \mu \mathrm{l}$, was also added in each library to confirm the reliability of

169 our method.

$170 \quad 2.2$ Microsatellite analysis

171 Multilocus genotypes were obtained by PCR amplification of 16 autosomal microsatellites

172 (Fleming et al., 1999; Cabria et al., 2007). The forward primer of each locus was 5'-end

173 labeled with a fluorescent dye. The following three multiplex sets were designed: mix 1

174 (Mvi 114, MLUT 25, MLUT 27, Mvis 099, Mvi 4001), mix 2 (Mvi 087, MLUT 32, MLUT

175 35, Mvis022, Mvi 1341) and mix 3 (MLUT 04, MER009, Mvis075, Mvis072, MER41,

176 MER022). PCR and genotyping steps were carried out following Pigneur et al. (2019).

177 Length variation determination (alleles and genotypes) was performed using Genemapper

1784.0 (Applied Biosystems). To construct consensus multilocus genotypes, an allele was only

179 accepted if observed at least twice. We thus accepted heterozygous genotypes that were

180 observed twice. A homozygote was accepted after three positive PCRs gave the same 181 single allele. 
182 The genetic structure of both sampled captive breeding centers was inferred using Bayesian

183 clustering analysis with Structure 2.3 software (Pritchard et al., 2000). We ran 10 iterations

184 for each $\mathrm{K}$ value from 1 to 5 using the admixture model. A total of $10^{6} \mathrm{MCMC}$ repetitions

185 were performed after a burn-in period of $20 \%$. The results of the 5 iterations for each $\mathrm{K}$

186 value were summarized and averaged using the Clumpp method (Jakobsson \& Rosenberg,

187 2007). The optimal number of clusters was investigated using the $\Delta \mathrm{K}$ method (Evanno et

188 al., 2005). F-statistics (pairwise FST, DJost and $\mathrm{F}_{\text {is }}$ ), allelic richness (Ar), the expected (He)

189 and observed (Ho) heterozygosity, as well as inbreeding coefficient (F) and multilocus

190 heterozygosity (MLH) were calculated for each defined group/individual in R version 3.5.2

191 (R Development Core Team, 2008). A Rst genetic distance estimation matrix between

192 individuals was generated using GenAlex 6.5 (Peakall \& Smouse, 2006).

1932.3 Amplification, sequencing and analysis of MHC genes

194 The fragment of DRB gene from exon 2 of MHC class II (Beta 1, 231 bp) was targeted 195 using the primers designed by Becker et al. (2009) for Mustela lutreola and a fragment 196 from exon 2 (alpha 1) from MHC class I, using the primers Meme-MHC-Iex2F and 197 PpLAa1L250 designed by Sin et al. (2012) for mustelids. PCRs were carried out in $25 \mu 1$ 198 volumes containing $0.9 \mu \mathrm{l}$ of primer mix, $5 \mu \mathrm{l}$ of GoTaq reaction buffer (Promega), $2 \mu 1$ of $199 \mathrm{MgCl}_{2}, 0.04 \mu \mathrm{l}$ of BSA, $0.8 \mu \mathrm{l}$ of dNTPs, $0.125 \mu \mathrm{l}$ of GoTaq G2 DNA polymerase 200 (Promega, France) and $3 \mu \mathrm{l}$ of DNA. The specific protocol was used for PCR: annealing 201 with touchdown protocol from $65^{\circ} \mathrm{C}$ to $56^{\circ} \mathrm{C}$ for $30 \mathrm{~s}$. Amplified DNA in duplicates were 202 pooled after quantification using the Quant-iT PicoGreen dsDNA Assay Kit (Thermo 203 Fisher Scientific Inc., Austria). The library preparation and sequencing were performed by 204 Novogene (UK). Using their designated library protocol, $2 \times 250$ bp paired-end sequencing 
205 with a depth of 50,000 reads/sample for MHC genotyping and was completed using an

206 Illumina NovaSeq platform (Illumina Biotechnology Co., Novogene, UK).

207 To analyze MHC-I and MHC-II amplicon sequences, we used the three-step pipeline

208 AmpliSAS (Sebastian et al., 2015). Low-quality sequences with Phred scores lower than

20920 were removed and clustering was conducted using the default parameters for Illumina

210 sequences. Already identified alleles of MHC-II DRB for E-mink were extracted from

211 NCBI (Becker et al., 2009), as well as sequences from related species (Mustela putorius

212 and Mustela itatsi) for MHC-I exon 2. If NCBI blast reveled $100 \%$ of sequence identify

213 between the discovered alleles in this study and already identified one, their name was

214 replaced by the accession number of these sequences. For the subsequent analysis, we

215 focused on the amino acid translated sequences (referred as MHC motifs) as they are in

216 direct contact with bacteria. We measured motif richness as the number of sequences per

217 individual for each locus. We calculated functional distances between individuals

218 following the approach described in Strandh et al. (2012). A maximum-likelihood tree was

219 constructed based on the chemical binding properties of the amino acids, as described by

220 five physico-chemical descriptor variables (z-descriptors) for each amino acid, using

221 sequences of Meles meles, Meles leucurus, Meles anakuma and Martes zibelina as out-

222 group retrieved through NCBI blast (Figure S1). The trees were used as reference from

223 which the functional distances between individuals were calculated using unweighted

224 UniFrac for both genes (Lozupone \& Knight, 2005). Following Bolnick et al. (2014), the

225 genetic distance between each amino acid sequences within each individual (Faith's PD)

226 were calculated, and further defined as motif divergence.

227 2.5 Statistical analysis for MHC and microsatellites markers between populations 
228 For subsequent analyses, individuals were sorted according to their population origin

229 (western and eastern) that corresponded to structure results (admixed individuals $(\mathrm{q}<0.9)$

230 were excluded $=0$ individuals). Non-parametric Kruskal-Wallis rank sum tests were used

231 to investigate neutral genetic variation with F and MLH calculated from microsatellite

232 markers analysis, between the two E-mink populations and host sex. Adaptive genetic

233 diversity and variation between E-mink populations and individual sex was also observed

234 using the same approach for motif richness for both MHC genes and divergence.

235 Permutational multivariate analyses of variance (PERMANOA) models adonis from the

236 vegan package were constructed with 9,999 permutations with reported $\mathrm{F}$, R2, and p-

237 values, to determine whether there were significant differences in genetic distance matrices

238 for neutral and adaptive markers between E-mink population, individual sex and birth

239 location. Pearson's pairwise correlation tests between the presence of MHC motifs, present

240 in at least three individuals, were conducted to potentially represent haplotype blocks for

241 the two genes investigated. The significance cutoff was set to p-value $<.05$ for each test.

242 Mantel tests were also conducted between each neutral, MHC-I and MHC-II genetic

243 distance matrices with 1,000 permutations to investigate correlation between each marker.

2442.6 Microbiota data generation and processing

245 After DNA extraction, the targeted gene for bacterial taxonomic affiliation using broad

246 bacterial primers of the region V4 of the 16S rRNA gene (515F-806R, $390 \mathrm{bp}$ ) was

247 amplified through PCRs. Amplification, library preparation and sequencing were carried

248 out in a similar manner to MHC genotyping, with a depth of 100,000 reads/samples of two

249 libraries composed of 48-52 samples. 
250 The quality controls of the demultiplexed paired-end reads were performed through the

251 software FastQC (Andrews, 2010). Demultiplexed sequence reads denoising and amplicon

252 sequence variants (ASVs) picking steps were done with the QIIME2 tool (Bolyen et al.,

253 2018; v. 2019.1), using the DADA2 pipeline (Callahan et al., 2016; Callahan et al., 2017).

254 Samples were pooled by individuals to limit bias from diet foods prior to rarefaction.

255 Rarefaction was conducted at 27,000 reads/samples in sampling depth. ASVs-or also

256 referred to as bacterial phylotypes - were then screened to the 97\% 16S rRNA gene full-

257 length reference sequences from the Silva v.132 database (Pruesse et al., 2007) for

258 taxonomical association using the VSEARCH classifier implemented in QIIME2

259 (Bokulich et al., 2018). Sequence alignment and phylogeny building were conducted in

260 QIIME2 for the construction of UniFrac distance matrices.

2612.7 Statistical analysis for $\alpha$-diversity of gut bacteria according to host information

262 All statistical analyses were conducted using the phyloseq and microbiome packages for 263 manipulation of data. Total observed number of bacterial taxa, Chao1, Shannon's diversity

264 index and Faith's PD in each sample were used as metrics to measure the $\alpha$-diversity of gut

265 bacteria between samples. Chao1 characterizes the overall phylotype richness within a

266 host, Shannon's diversity index considers richness and abundance, and Faith's PD is an

267 indicator of genetic diversity within a sample. Differences in the indices according to E-

268 mink populations, sex and birth location of the host were analyzed using Kruskal-Wallis

269 rank sum tests. Linear regression models were also conducted with the different measures

270 of the microsatellites and MHC analysis (MLH, F for microsatellite data, MHC motif

271 richness and divergence) as predictors, and microbial richness indexes as response variable. 
272 Homogeneity of variance assumptions were tested using Levene tests and normality of the

273 residuals with Shapiro-Wilk tests and visual representations.

2742.8 Statistical analysis for $\beta$-diversity of gut bacteria between population and differential

275 abundance

276 Weighted and unweighted UniFrac distance matrix between samples were used to

277 investigate differences in gut microbial communities between E-mink population and host

278 sex. These metrics consider the differences in phylogenetic distance and abundance of each

279 bacterial community between samples, pairwise, emphasizing on rare (unweighted) or

280 abundant microbial taxa (weighted). PERMANOVA tests were conducted in similar

281 conditions than PERMANOVA tests conducted on MHC matrixes. Additionally, models

282 were constructed with adaptive genetic variables: motif richness and divergence from both

283 MHC genes. To test the hypothesis that both neutral and adaptive distances are correlated

284 with gut microbial composition, we employed Mantel tests between each genetic distance

285 and Unifrac distance.

286 A differential abundance analysis was conducted on the raw ASVs count (after filtering,

287 prior to rarefaction) that were present in more than $10 \%$ of all the samples and that had a

288 relative abundance of more than 5\% among all taxa. It corresponds to the core microbiota

289 of the dataset, represented by 1203 phylotypes. The abundance analysis was made at the

290 ASV level with the DESeq2 package, using a negative binomial Wald test to test

291 significance in contrast between each E-mink population and each common MHC motifs

292 that were present in at least two individuals. Only microbial ASV with a significance level

$293(\alpha)$ below .001 after false discovery rate (FDR) corrections were considered using the

294 Benjamini-Hochberg method (Love et al., 2014). From the same core microbiota, we also 
295 tested for Pearson correlations between microbial genera' relative abundance per

296 individual and continuous genetic variables (MLH, motif richness and PD for both MHC

297 class I and II) with FDR corrections, for genera that were encountered at least in three

298 individuals.

\section{Results}

3003.1 Genetic diversity of the European mink captive populations

301 The microsatellite markers analysis demonstrated an overall population allelic richness per

302 locus of 2.69 with an average of 2.49 in the western population, and 2.82 in the eastern

303 population. Heterozygosity values were lower in the western population compared to

304 eastern population (Table 1). Bayesian assignment recovered two genetic clusters within

305 our population, and no admixture pattern were detected. All individuals clustered according

306 to populations, corresponding to the two different breeding facilities. The offspring with

307 parents of each population was assigned to the western population according to clustering

$308\left(\mathrm{p}\left(\mathrm{K}_{\text {western }}\right)=0.975\right)$. Multilocus heterozygosity was slightly higher in eastern than western

309 populations (Kruskal-Wallis: $\chi^{2}=3.4761$; $p$-value $=0.0623$ ), but the inbreeding coefficient

310 (F) was not (Kruskal-Wallis: $\chi^{2}=0.085714$; p-value=0.7697). Overall, sex and birth

311 location had no significant effect on neutral markers' diversity and richness.

312 PERMANOVA on genetic distance based on the microsatellite markers detected no

313 variation according to E-mink population, sex and birth location (Table 2).

314 Raw MHC amplicon sequencing data consisted of 3,084,478 raw reads with an average

315 length of 230 base pairs for MCH-I and 130 base pairs for MHC-II. After processing, we

316 characterized 13 MHC-I motifs (amino acid sequences) and 6 MHC-II motifs. The average

317 number of motifs per individual was 5.3 and 3.08 (range: 2-9; 2-4) for MHC-I and MHC- 
318 II genes respectively, indicating the presence of at least five and two copies for the two

319 regions. For the MHC-I gene, three motifs were strictly present in the eastern E-mink, and

320 one motif in the western E-mink. Comparatively, no motif were unique to eastern E-mink

321 for MHC-IIex2 gene, and three were strictly found in western E-mink (Figure 1). Spearman

322 correlation tests allowed us to detect haplotype blocks for both genes, mostly attributed to

323 the eastern population (with the motifs Mulu:MHC-I*0003, Mulu:MHC-I*0008,

324 Mulu:MHC-I*0012, Mulu:MHC-I*0013 and Mulu:MHC-I*0015 for MHC-I and

325 Mulu:DRB*90701, EU263553 for MHC-II) and western E-mink (Mulu:MHC-I*0007,

326 Mulu:MHC-I*0009 and Mulu:MHC-I*0011 for MHC-I, KM371114_EU263551,

327 EU263558_LC055119, EU263550_EU263557 and EU263554_EU263552_EU263556 for

328 MHC-II, Figure S2 \& Table S2). Most of the variation encountered in both genes was

329 expressed in amino acid residues that influence the binding of CD4 and CD8 glycoproteins

330 involved in antigen presentation for adaptive immunity (Figure S3).

331 Motif richness and divergence (Faith's PD) were significantly higher in the western E-

332 mink compared to eastern E-mink for MHC-II gene (Kruskal-Wallis: $\chi^{2}=13.456$, p-

333 value $=0.0002 ; \chi^{2}=8.0614, \quad \mathrm{p}$-value $=0.0045 ;$ respectively). However, for MHC-I,

334 divergence was higher in eastern E-mink compared to western E-mink, but not motif

335 richness (Kruskal-Wallis: $\chi^{2}=5.0097, \quad \mathrm{p}$-value $=0.0252 ; \quad \chi^{2}=1.5456, \quad \mathrm{p}$-value $=0.2138$,

336 respectively). No changes in motif richness nor divergence were observed according to sex

337 for the two genes. However, we did observe significant variation in MHC-II richness

338 according to birth location (Kruskal-Wallis: $\chi^{2}=10.854$, p-value $=0.0125$ ), and a Dunn test

339 with Benjamini-Hochberg correction only detected higher motif richness for the MHC-II

340 gene in captive-born E-mink in Spain compared to the EEP (Dunn: Z=-2.748, adjusted p- 
341 value=0.0358). PERMANOVA detected a significant influence of E-mink sex for MHC-I

342 genetic distance, as well an influence of mink population close to the significance threshold

343 (Figure S5), whereas E-mink population was the only variable influenced MHC-II

344 composition variation (Table 2). Finally, Mantel tests showed a positive correlation

345 between MHC - I and neutral markers distances (Mantel: r=0.2761, p-value=0.001).

$3463.2 \alpha$-diversity of gut bacteria according to host information

347 A sample of mock community containing known concentrations of genomic DNA from 20

348 bacterial strains was sequenced. 19 of the 20 different strains originally included in the

349 sample were detected. The undetected strain was present at the lowest concentration.

350 Therefore, our protocol allowed bacterial DNA detection and identification to the genus

351 level as long as its concentration in the DNA extract was at least $2.8 \mathrm{pg} / \mu \mathrm{l}$, and provided

352 that the sequence was included in the reference database.

353 After reads processing, a total read count of 624,796 was obtained for gut microbial

354 communities in captive E-mink, with an average counts per sample of 26,033 after

355 rarefaction to limit sequencing depth artifacts. A total number of 5703 ASVs - or

356 phylotypes - were distinguished in the samples. The gut microbiota of the E-mink was

357 mostly composed of the Firmicute phylum (74\%), which was dominated by the

358 Clostridiaceae and Peptostreptococcaceae families, followed by Proteobacteria (14\%)

359 with Enterobacteriaceae, Moraxellaceae and Pseudomonadaceae families (Figure 2).

360 Despite an overall observation of lower microbial richness in the western compared to the 361 eastern populations, no significant results were observed in multiple microbial richness

362 indices (Shannon index, Chao1; Figure 3). However, we did observe slightly lower Faith's

363 PD in western compared to eastern individuals $\left(\chi^{2}=2.8834\right.$, p-value $\left.=0.0895\right)$. Western 
364 females had significantly lower microbial phylogenetic diversity compared to males

365 (Figure 3). Despite not reaching statistical significance $\left(\mathrm{R}^{2}=0.1626, \mathrm{~F}=0.5502\right.$, $\mathrm{p}$ -

366 value=0.7633), linear regression models with alpha diversity measures as response

367 variables showed negative correlations with adaptive genetic richness measures, and

368 particularly strong estimates for MHC-I richness (Figure S4).

$3693.3 \beta$-diversity of gut bacteria between E-mink and differential abundance

370 Beta diversity analyses revealed that bacterial communities were significantly different in

371 composition according to E-mink population only when considering unweighted Unifrac

372 distances, whereas no significant differences in microbial community composition were

373 found between host sexes nor birth locations (Models 4 and 6, Table 2). Moreover, MHC-

374 II gene richness had a small significant influence on gut microbial composition (Model 5,

375 Table 2). This is reflected in the results of the PCoA, which clustered individuals with

376 differences in number of MHC-II motifs (Figure 4). Mantel tests also shown a significant

377 positive correlation between unweighted Unifrac distance and MHC-II genetic distance

378 (Mantel: $\mathrm{r}=0.4811$, $\mathrm{p}$-value=0.019), and despite not reaching statistical significance, a

379 negative correlation with MHC-I genetic distance (Mantel: r=-0.0823, p-value=0.862) and

380 close to zero for neutral markers' distance (Mantel: $r=0.0065$, $p$-value=0.229).

381 We recovered several bacterial genera and families whose relative abundances were

382 significantly correlated with MHC-I and MHC-II richness and divergence, while some

383 marginally correlated with multilocus heterozygosity of neutral markers (Table 3). A

384 majority $(65 \%)$ of the Pearson correlations appeared to be negative between bacterial

385 genera and genetic indexes. Differential abundance analysis in microbial families

386 according to presence of MHC motifs for both genes between E-mink populations detected 
387 a significant increase in abundance for 13 families in the eastern population, for 8 in 388 western E-mink (Figure 5).

389 From the differential abundance analysis, 22 phylotypes were found to be significantly 390 different in abundance according to MHC motifs presence. MHC-I motifs explained the 391 variation of 3 phylotypes, and only impacted the Clostridiaceae family (Clostridium sensu 392 stricto 1). MHC-II motifs presence was attributed to the altered the abundance of 14 393 microbial phylotypes and those were mostly more abundant in the eastern E-mink as 13 394 phylotypes were more abundant in eastern E-mink against one in the western E-mink. 395 MHC-II motifs impacted over twelve genera compared to one for MHC-I motifs. It is also 396 worth noting that both MHC-I and MHC-II motifs presence were observed to alter taxa 397 abundance for 5 phylotypes, mostly from the Proteobacteria phylum (Table 4). The MHC398 II motifs KM371114_EU263551, EU263554_EU263552_EU263556, and 399 Mulu:DRB*90701 respectively took part in the variation in abundance for 17, 15 and 12 400 phylotypes respectively. MHC-I motif Mulu:MHC-I*00008 was significantly involved in 401 the variation for 4 phylotypes, whereas the other motifs had relatively low impact, as they 402 took part in abundance variation of 1-2 families.

403

\section{Discussion}

405 Genetic variation in the two E-mink captive breeding programs

406 Microsatellite markers for both populations exhibited low allelic richness and 407 heterozygosity indices, with the western population having the lowest values, in line with 408 previously published results (Michaux et al., 2005; Cabria et al., 2007; Cabria et al. 2015).

409 However, the eastern E-mink sampled in this study might not reflect the full genetic 
410 variation within the entire population, as collected E-mink originated from a subset of the

411 EEP since its start 25 years ago (Becker et al., 2009). Conversely, western E-mink sampled

412 came from wild-born and captive-born individuals from a recent breeding program. Our

413 Bayesian clustering analysis suggests the existence of at least two main genetic units of E-

414 mink defined by their origin with the captive programs, validating our use of the two E-

415 mink groups for studying their genetic and gut microbial variation.

416 Nonetheless, the two MHC genes investigated revealed differential variation between the

417 two E-mink populations, the MHC-I gene being more divergent in eastern E-mink and the

418 MHC-II gene exhibiting more richness and divergence in western E-mink. Interestingly,

419 the adaptive genetic diversity followed the neutral markers trend only for one gene and not

420 the other, making the assessment of genetic diversity in captive breeding complex. The

421 maintenance of genetic variation in neutral markers through non-selective evolutionary

422 forces (genetic drift, inbreeding) depend on the number of founders in a population, as well

423 as the breeding system of the species. However, balancing selection is believed to

424 counteract those non-selective evolutionary forces in functional genes (Hedrick, 1999),

425 resulting in an excess of heterozygotes in small, isolated populations for MHC-II loci. This

426 pattern has been observed in several isolated populations (Aguilar et al., 2004; Jarvi et al.,

427 2004; Schad et al., 2004), but all species investigated were free ranging, implying less

428 restrictions in the mating system compared to CBPs and therefore stronger sexual selection.

429 In line with previous evidence of the role of sexual selection for MHC pattern distribution

430 in vertebrates (Edwards \& Hedrick, 1998), we observed that sex had an influence on MHC-

431 I gene composition. It has been shown that MHC class I genes may be involved in 432 pheromone recognition, and that mate preferences can be reflected in dissimilarity of MHC 
433 patterns (Penn, 2002). In the case of the E-mink, captive-bred males are less successful

434 breeders compared to wild-born males (Kiik et al., 2013). Therefore, mate pairing based

435 only on pedigree might not provide enough information and might be hindered by MHC-I

436 similarities between potential mates. Variation at neutral markers may thus not accurately

437 reflect variation at potentially relevant genes, particularly those under selection like the

438 MHC (Ujvari \& Belov, 2011), and a global genetic assessment should be taken in

439 consideration in conservation genetics for management decisions (Mardsen et al., 2013).

440 The ecosystem on a leash model in mammalian gut microbiota

441 The hypothetical framework presented by the ecosystem on a leash model (Foster et al.,

442 2017) suggests that more host control in distantly related microbes, illustrated by the

443 Faith's PD index and Unifrac distances, should be found in individuals with high MHC

444 diversity. This pattern was observed, although weakly, in alpha diversity analysis for the

445 western population, highlighting the importance of the MHC class II gene above MHC

446 class I and microsatellite markers. It is also worth noting that this result only involves

447 distantly related microbes, emphasizing the fact that a phylogenetically diverse microbiota

448 could lead to the dominance of the fastest growing microbes instead of the microbes that

449 are most beneficial to the host, lending support to the ecosystem on a leash model.

450 Similar more robust trends were found in beta-diversity analysis, where gut microbial 451 composition was different according to the number of MHC-II motifs a E-mink possessed

452 and the more distant two E-mink are in MHC-II haplotype, the more different in rare gut 453 microbiota composition as well. Mostly negative correlations were observed between 454 microbial abundance and MHC genes richness and divergence, likewise suggesting more 455 host control in individuals with high adaptive genetic variation. This also supports an 
456 advantage in balancing selection despite strong genetic drift. The differential abundance

457 analysis also revealed stronger host control in the western population, mostly explained by

458 the presence of specific MHC-II motifs. Moreover, the MHC-II gene was more likely to

459 impact a wide range of microbial taxa. These results are in line with previous studies

460 conducted on fish, mice and birds (Bolnick et al., 2014; Khan et al., 2019; Leclaire et al.,

461 2018). However, we did observe that one motif of MHC-II present in the eastern population

462 also impacted the abundance of several bacterial families, indicating that the captive

463 eastern population still possess interesting motifs for host control.

464 Because the MHC-I gene targets intracellular non-self-molecules recognition, it would

465 impact a smaller number of bacteria compared to MHC-II (Ost \& Round, 2018). Other

466 taxonomic groups such as viruses and protists would need to be targeted for further

467 investigation, and eastern population might be more equipped to recognize them, given the

468 increased diversity for this MHC-I gene (Kubinak et al., 2012). This is of particular

469 importance knowing the circulation of several viruses in free-ranging western E-mink

470 (Fournier-Chambrillon et al., 2004; Philippa et al., 2008; Mañas et al., 2016). The canine

471 distemper virus, which results in a high mortality rate in E-mink, is currently re-emerging

472 in many wild carnivore populations in Europe (Origgi et al., 2012) and has had a major

473 impact on population of E-mink in Navarra, Spain (Fournier-Chambrillon et al., 2022).

474 Overall, our prediction that less host control will be observed in mink with lower genetic

475 diversity is supported by both alpha and beta diversity for the E-mink. However, both 476 populations have low genetic diversity, and the MHC class II DRB gene seemed to have a

477 stronger influence in gut microbes than other markers. To further validate our results,

478 replicating the study to see if those differences are observable when individuals from the 
479 two populations are kept in the same facility to control for the influence of the external

480 environment should be conducted. Given that we only had access to samples from a small

481 fraction of the captive eastern population, our results might also not be representative of

482 the entire captive breeding stock. Despite the gut microbiota variation being a complex

483 puzzle, our study gives more importance to host immunogenetics in the context of species

484 conservation.

485 Adaptation to captivity and management practices

486 For MHC genes, rare allele and heterozygous advantage are two types of balancing

487 selection that have been suggested to be important in maintaining high levels of adaptive

488 genetic diversity (Sommer, 2005). Assuming that rare and divergent MHC genotypes are

489 more likely to induce host control on gut microbes, giving a fitness advantage to the host,

490 the co-evolutionary arm race with gut microbes will foster adaptation from microorganisms

491 to not be targeted by common MHC alleles (Kubinak et al., 2012). However, microbe-

492 driven selection could vary over time and space and between E-mink populations. This

493 mechanism could be of influence in the western E-mink population, given that the breeding

494 program started in 2013 and individuals from the wild are still being captured to increase

495 founder size in the program from the natural habitat. Moreover, low MHC class II gene

496 diversity in the eastern population might indicate that non-evolutionary forces overshadow

497 balancing selection for this locus, which could be mainly explained by extensive constrains

498 in the mating system for a long period of time.

499 Inadvertent genetic adaptation to captivity for endangered species has been documented

500 over recent years (reviewed in Frankham, 2008). This has been related to a fitness reduction

501 when animals are released in the wild environment, increasing with the numbers of captive- 
502 bred generations, including changes in reproductive success, morphology and behavior

503 (Williams \& Hoffman, 2009; Willoughby \& Christie, 2019). Becker et al. (2009)

504 previously investigated the MHC-II DRB gene in the captive eastern E-mink population,

505 and detected nine alleles, representing 6 motifs. However, ten years later, we observed 3

506 motifs in the eastern group. The EEP in Estonia started in 1992 and has not been

507 supplemented by wild individuals for at least 25 generations (T. Maran, pers. comm.,

508 2021). Moreover, given the moderate success of the breeding program due to captive-born

509 male behavior (Kiik et al., 2013), this suggests that high number of generations in captivity

510 led to loss of genetic diversity and deleterious genetic fixation took place for this

511 population (Woodworth et al., 2002; Frankham, 2008; Witzenberger \& Hochkirch, 2011;

512 Parmar et al., 2017). Even though $90 \%$ of the initial gene diversity has been maintained

513 through studbook calculations (T. Maran, pers. comm., 2021), it is likely that studbook

514 measurements might not reflect this trend for all E-mink genes. However, different

515 management strategies have been proposed to mitigate fitness reduction for future

516 reintroduction (reviewed in Williams \& Hoffman, 2009) that could be implemented for the

517 E-mink.

518 One strategy is to translocate animals between breeding centers for reproduction to prevent

519 loss of genetic diversity. Similar to the western captive population of E-mink, these

520 translocations could be composed of wild-born individuals, free of captive selection

521 pressure (Schulte-Hostedde \& Mastromonaco, 2015). Occasional translocations from

522 western to eastern captive populations could also be conducted and would potentially

523 mitigate the modest reproductive success within the program. It is worth noting that wild-

524 born animals have been out of reach from the EEP breeding stock so far. However, 
525 conducting preliminary MHC variation assessment on reintroduced animals from the

526 eastern stock present in Hiiumaa island, as they no longer face captivity for a number of

527 generations, could be used to identify potential assets to the current breeding stock.

528 Captivity has been shown to alter gut microbial communities (McKenzie et al., 2017).

529 Combined with this traditional conservation efforts, microbial rescue could also help

530 improve success of managing at-risk populations. For example, the most common cause of

531 mortality in captive cheetahs (Acinonyx jubatus) is bacterial infection, possibly because of

532 an increase in pathogenic taxa compared to wild conspecifics (Wasimuddin et al., 2017).

533 Microbial rescue, using probiotics, can stabilize the composition of the gut microbiota of

534 dolphin in captivity (Lagenorhynchus obliquidens, Cardona et al., 2018). Implementing

535 wild-like diet-based enrichment could also mitigate captivity effects on gut microbial

536 communities in the same way as captive selection (Mueller et al., 2019; Trevelline et al.,

537 2019; van Leeuwen et al., 2020), but further research is needed in the E-mink case.

538 These types of strategies could increase adaptive genetic diversity related to

539 immunomodulation and therefore a fitness advantage to the mink once reintroduced.

540 Coupled with a more in-depth investigation on the gut microbiota of the E-mink according

541 to diet and environment manipulation, these technics can have synergetic effects and foster

542 the success of the CBPs (Gould et al., 2018; West et al., 2019). This first look into the

543 connection between management strategies, genetic diversity and gut bacteria within the

544 CBPs of the E-mink allowed preliminary assessment of the current situation. It also offers

545 many axes of further research and potential strategies with the on-going challenges that

546 many ex situ conservation programs face to mitigate species extinction. 


\section{Declarations}

549 Funding: Funding was supported by the NSERC CREATE grant, ReNewZoo and

550 Conversation Genetics Laboratory of the University of Liège

551 Conflict of interest: All authors certify that they have no affiliations with or involvement

552 in any organization or entity with any financial interest or non-financial interest in the

553 subject matter or materials discussed in this manuscript.

554 Data availability: Supporting information has been made available online. Pooled 16S

555 rRNA sequences available on NCBI accession number: SUB9956843. ASV table,

556 taxonomy table and mapping file have been uploaded, as well as MHC-1, MHC-2 and

557 microsatellite data: Dryad link XXXX, see reference list.

558 Authors' contributions: J.M., A.S.H. and P.V.L. planned and designed the study. P.V.L.

559 with the help of C.F.-C., P.F., C.A. and L.B. performed the sample preparation for

560 sequencing. J.M. provided sequencing services and together with A.S.H. advised on

561 laboratory and sampling procedures. P.V.L. performed bioinformatics, statistical analyses

562 and the interpretation of results with feedbacks provided by A.S.H. and J.M.. P.V.L. wrote

563 the manuscript with input from all authors.

564 Acknowledgments: FIEB (Ciprian Petrescu), Madis Podra, OFB (Christelle Bellanger,

565 Maylis Fayet), Zoodyssée (Mathilde Picard, Suzon Berton, Guillaume Romano), Lise

566 Marie Pigneur, Alice Mouton, Adrien André, EEP program for the European mink, Tiit

567 Maran and the Ministry for the Ecological Transition and the Demographic Challenge 568 (Spain).

569 Tables and figures 


\begin{tabular}{|l|c|c|lc}
\cline { 2 - 5 } \multicolumn{1}{c|}{} & Eastern & Western & Distance between populations \\
\hline $\mathrm{N}$ & 14 & 10 & Gst & 0.0615 \\
$\mathrm{~A}$ & 50 & 43 & Djost & 0.0284 \\
$\mathrm{Ar}$ & 2.82 & 2.49 & Fst & 0.1165 \\
& 0.54 & 0.44 & & \\
$\mathrm{He}$ & 0.48 & 0.43 & & \\
$\mathrm{Fis}$ & -0.1241 & -0.0403 & & \\
Fis_Low & -0.252 & -0.2265 & & \\
Fis_High & -0.0177 & 0.1047 & &
\end{tabular}

571 Table 1. Measures of neutral genetic diversity through microsatellite marker analysis by E-

572 mink captive population $($ Eastern $=$ EEP breeding center in Zoodysée, France; Western $=$ 573 FIEB breeding center in Spain). N: number of individuals, A: total number of alleles, Ar: 574 mean allelic richness per locus, Ho: observed heterozygosity, He: expected heterozygosity, 575 Fis: mean inbreeding coefficient. Overall mean value for each population across the 16 576 markers. 
578

\begin{tabular}{|c|c|c|c|c|c|}
\hline Model & Distance matrix & Variables & F statistic & $\mathrm{R}$ square & P-value \\
\hline 1 & Microsatellite & $\begin{array}{l}\text { Mink population } \\
\text { Birth location } \\
\text { Sex } \\
\text { Residuals }\end{array}$ & $\begin{array}{l}1.45837 \\
1.42653 \\
0.77162\end{array}$ & $\begin{array}{l}0.06286 \\
0.06149 \\
0.09978 \\
0.77587 \\
\end{array}$ & $\begin{array}{l}0.2318 \\
0.2390 \\
0.7236\end{array}$ \\
\hline 2 & MHC-I & $\begin{array}{l}\text { Mink population } \\
\text { Birth location } \\
\text { Sex } \\
\text { Residuals }\end{array}$ & $\begin{array}{l}2.8442 \\
0.9910 \\
5.1459\end{array}$ & $\begin{array}{l}0.09820 \\
0.10264 \\
0.17767 \\
0.62148 \\
\end{array}$ & $\begin{array}{l}0.0797 \\
0.4476 \\
\mathbf{0 . 0 2 0 0 ( 0 . 3 8 7 )}\end{array}$ \\
\hline 3 & MHC-II & $\begin{array}{l}\text { Mink population } \\
\text { Birth location } \\
\text { Sex } \\
\text { Residuals } \\
\end{array}$ & $\begin{array}{l}\mathbf{5 . 4 9 8 4} \\
0.7741 \\
0.2503\end{array}$ & $\begin{array}{l}\mathbf{0 . 2 1 0 9 0} \\
0.08908 \\
0.00960 \\
0.69042 \\
\end{array}$ & $\begin{array}{l}\mathbf{0 . 0 2 8 4 ( 0 . 4 1 8 )} \\
0.5412 \\
0.7140\end{array}$ \\
\hline 4 & Microbiota (UWU) & $\begin{array}{l}\text { Mink population } \\
\text { Birth location } \\
\text { Sex } \\
\text { Residuals } \\
\end{array}$ & $\begin{array}{l}1.44739 \\
0.96248 \\
0.99747\end{array}$ & $\begin{array}{l}\mathbf{0 . 0 6 2 0 3} \\
0.12375 \\
0.04275 \\
0.77146 \\
\end{array}$ & $\begin{array}{l}\mathbf{0 . 0 0 0 3}(\mathbf{0 . 2 2 9 )} \\
0.7692 \\
0.4722\end{array}$ \\
\hline 5 & Microbiota (UWU) & $\begin{array}{l}\text { MLH } \\
\text { MHC-II divergence } \\
\text { MHC-Il richness } \\
\text { MHC-I divergence } \\
\text { MHC-I richness } \\
\text { Residuals } \\
\end{array}$ & $\begin{array}{l}0.97674 \\
1.06218 \\
1.21292 \\
0.99014 \\
1.10666\end{array}$ & $\begin{array}{l}0.04194 \\
0.04561 \\
\mathbf{0 . 0 5 1 9 5} \\
0.04252 \\
0.04752 \\
0.77292 \\
\end{array}$ & $\begin{array}{l}0.5673 \\
0.2064 \\
\mathbf{0 . 0 2 1 9}(\mathbf{0 . 8 2 1 )} \\
0.5019 \\
0.1151\end{array}$ \\
\hline 6 & Microbiota (WU) & $\begin{array}{l}\text { Mink population } \\
\text { Birth location } \\
\text { Sex } \\
\text { Residuals }\end{array}$ & $\begin{array}{l}1.26796 \\
0.40095 \\
0.62848\end{array}$ & $\begin{array}{l}0.06009 \\
0.05701 \\
0.02979 \\
0.85311 \\
\end{array}$ & $\begin{array}{l}0.2749 \\
0.8163 \\
0.6250\end{array}$ \\
\hline 7 & Microbiota (WU) & $\begin{array}{l}\text { MLH } \\
\text { MHC-II divergence } \\
\text { MHC-Il richness } \\
\text { MHC-I divergence } \\
\text { MHC-I richness } \\
\text { Residuals }\end{array}$ & $\begin{array}{l}1.24147 \\
0.27059 \\
1.37532 \\
0.57734 \\
1.05875\end{array}$ & $\begin{array}{l}0.05512 \\
0.01201 \\
0.06106 \\
0.02563 \\
0.04701 \\
0.79917\end{array}$ & $\begin{array}{l}0.2745 \\
0.7573 \\
0.2411 \\
0.6026 \\
0.3326\end{array}$ \\
\hline
\end{tabular}

Table 2. Model results of PERMANOVAs for 9,999 permutations for each distance matrix 582 according to variables of interest. Values in bracket are p-values from Marti Anderson's 
583 PERMDISP2 procedure for the analysis of multivariate homogeneity of group dispersions 584 (variances). UWU: Unweighted Unifrac, WU: Weighted Unifrac distances.

585

586

\begin{tabular}{|c|cc}
\hline Genus (family) & Pearson's rho & FDR p-value \\
\hline Multilocus heterozygosity (neutral markers) & & \\
Clostridium sensus stricto 13 (Clostridiaceae) & 0.365 & 0.065 \\
Epulopiscium (Lachnospiraceae) & 0.410 & 0.034 \\
Gemmela (Gemellaceae) & -0.352 & 0.077 \\
Providencia (Morganellaceae) & 0.367 & 0.063 \\
\hline MHCl ex2 richness & & \\
Helicobacter (Helicobacteraceae) & -0.400 & 0.040 \\
Staphylococcus (Staphylococcaceae) & -0.386 & 0.049 \\
\hline MHCl ex2 divergence (PD) & & \\
Aeromonas (Aeromonadaceae) & 0.382 & 0.065 \\
Helicobacter (Helicobacteraceae) & -0.436 & 0.033 \\
Paeniclostridium (Peptostreptococcaceae) & -0.384 & 0.064 \\
Pseudomonas (Pseudomonadaceae) & 0.473 & 0.019 \\
Staphylococcus (Staphylococcaceae) & -0.467 & 0.021 \\
\hline Actinomyces (Actinomycetaceae) & & \\
Luteolibacter (Rubritaleaceae) & -0.438 & 0.022 \\
Sporosarcina (Planococcaceae) & 0.366 & 0.064 \\
Streptococcus (Streptococcaceae) & 0.398 & 0.048 \\
MHCII ex2 richess & -0.395 & 0.043 \\
\hline Helicobacter (Helicobacteraceae) & & \\
Lysinibacillus (Planococcaceae) & -0.456 & 0.025 \\
Staphylococcus (Staphylococcaceae) & -0.632 & 0.0035 \\
Streptococcus (Streptococcaceae) & -0.540 & 0.046 \\
Terrisporobacter (Peptostreptococcaceae) & -0.711 & 0.0001 \\
Genta that Were signican & -0.516 & 0.063
\end{tabular}

588 Table 3. Genera that were significantly correlated with or differed across variables 589 associated with gut microbial communities. Italicized P-values were marginally significant 590 after False Discovery Rate (FDR) correction of p-values.

591

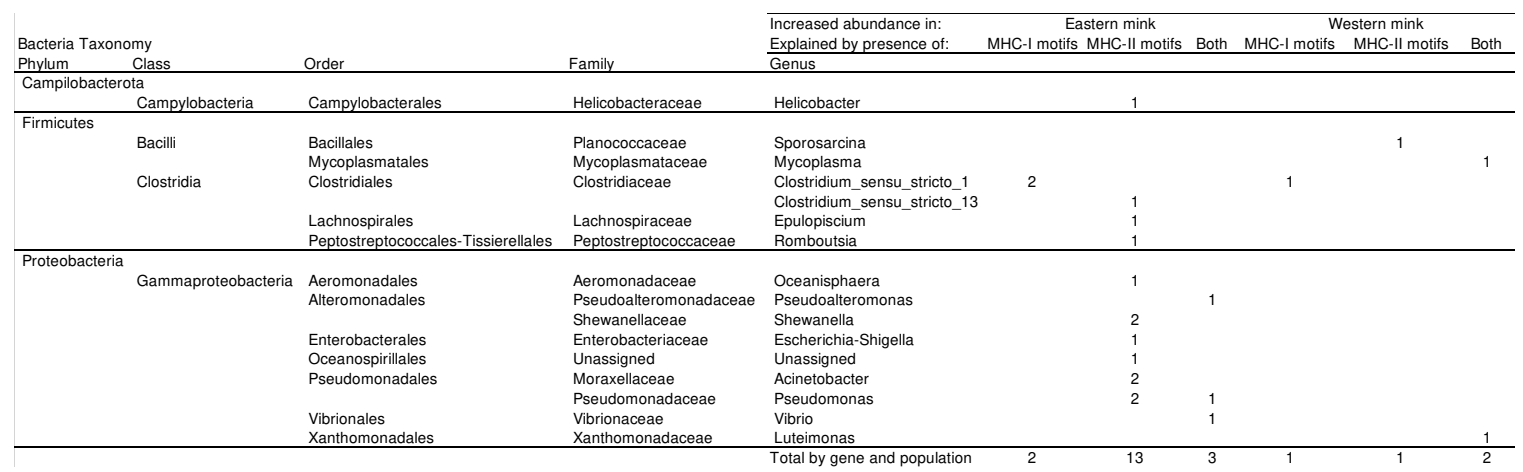

593 Table 4. Summary of significantly enriched phylotypes among the two mink group 594 according to presence of each MHC motif in each class I and class II genes from the 595 DESEq2 analysis. If multiple motifs explained the variation of one ASV, there were 596 accounted for once if it varied only according to motifs from the same gene. 


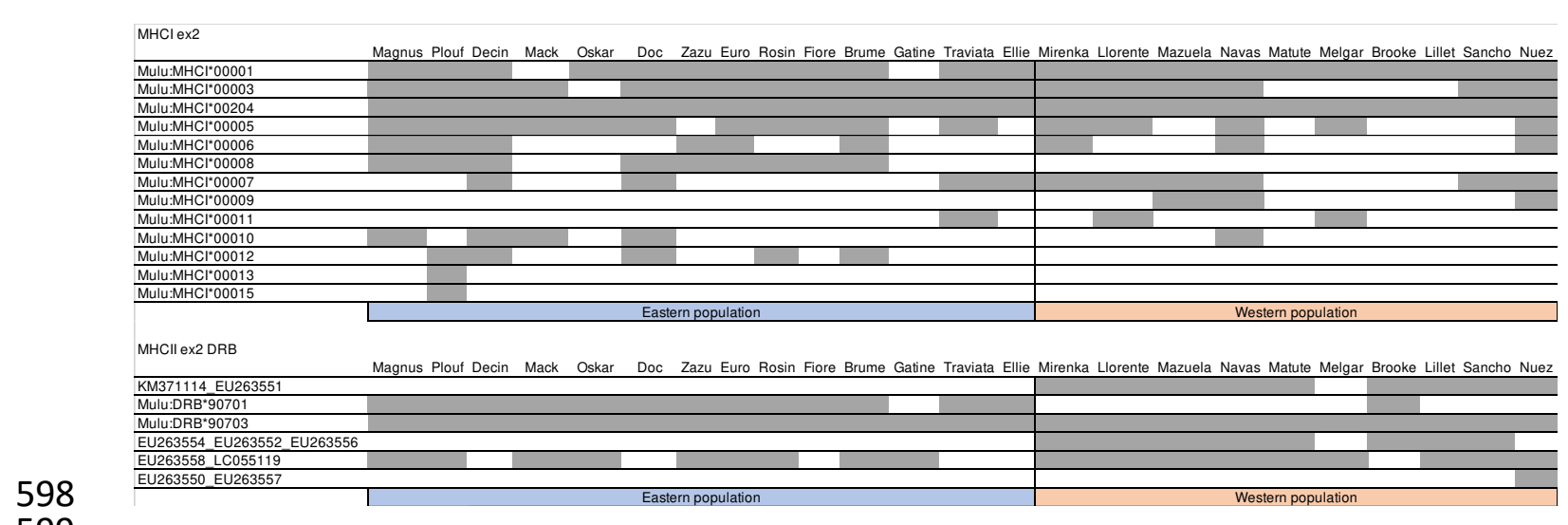

Figure 1. Distribution of MHC motifs in both mink population. Grey shading shows the 601 presence of each motif for each individual. Newly discovered motifs were named following 602 the nomenclature, and already identified motifs were named according to the accession 603 number found through NCBI blast.

604

605

606

607

608 609
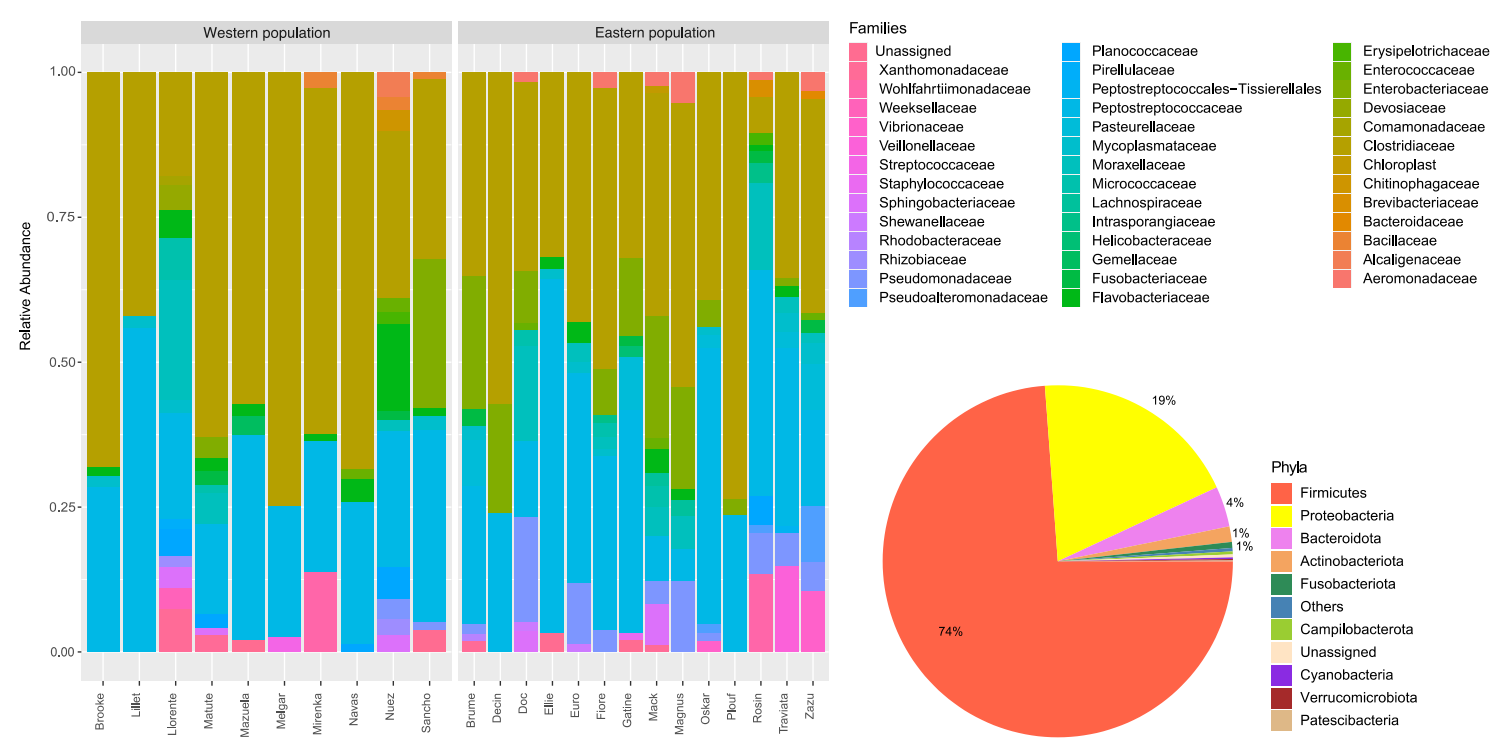

Figure 2. The relative abundance of common bacterial families across 24 E-mink individuals from the two breeding populations. The pie chart represents the mean proportion of relative abundance for each microbial phylum for all individuals. 

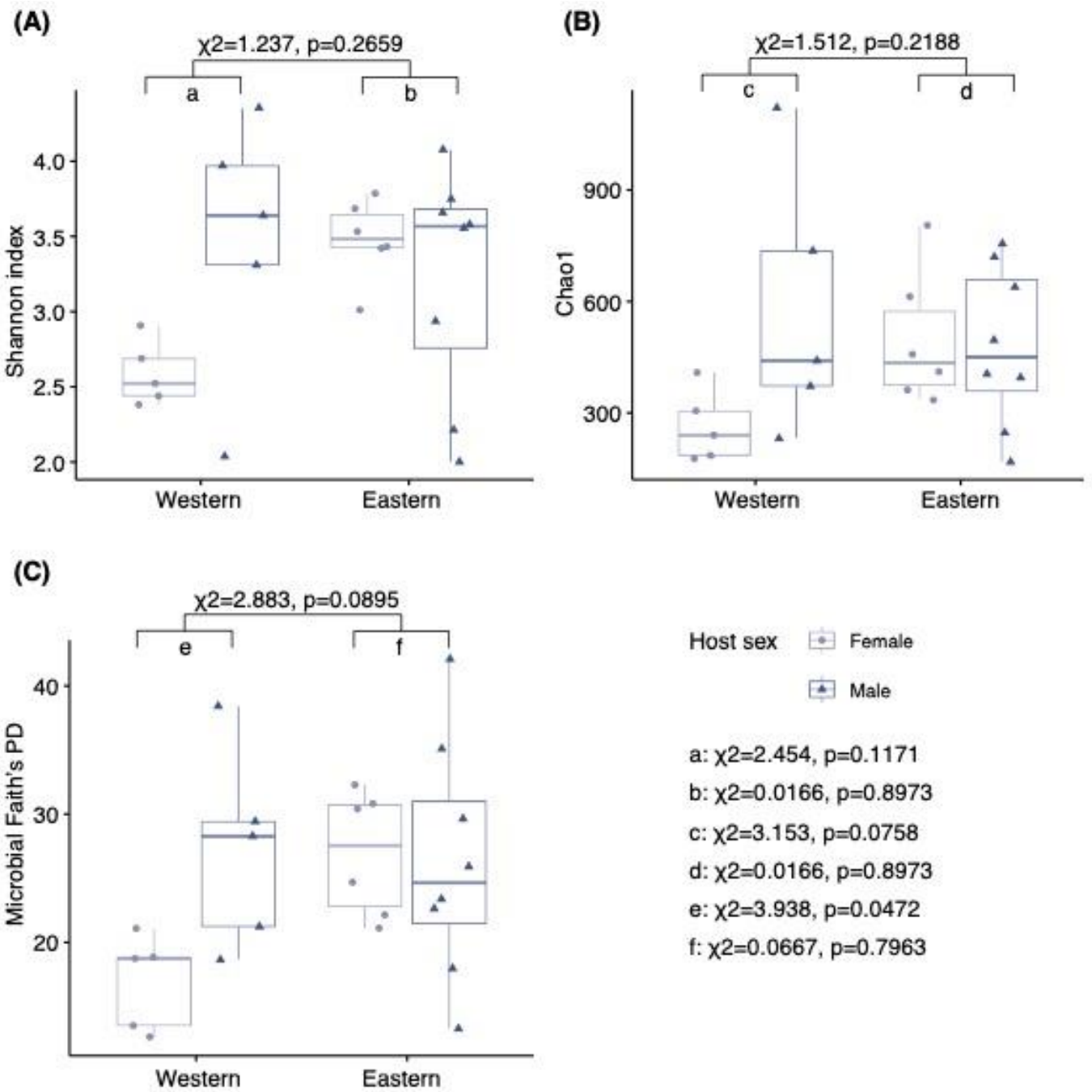

$$
\begin{aligned}
& \text { Host sex } \\
& \text { a: } \chi^{2}=2.454, p=0.1171 \\
& b: x^{2}=0.0166, p=0.8973 \\
& \text { c: } \chi^{2}=3.153, p=0.0758 \\
& d: \chi^{2}=0.0166, p=0.8973 \\
& \text { e: } \chi^{2}=3.938, p=0.0472 \\
& f: \chi^{2}=0.0667, p=0.7963
\end{aligned}
$$

Figure 3. Alpha diversity indexes for microbial taxa richness are shown as boxplots (with median, interquartile range (IQR) and whiskers extending to the last data points). Individual values appear as light blue dots for the female mink and dark blue triangle for the males, for each mink populations. Shannon index (A) expresses changes in taxa richness and abundance, Chaol (B) variation in strict richness, and Faith's PD (C) for phylogenetic diversity. Results from respective Kruskal-Wallis tests are documented. 


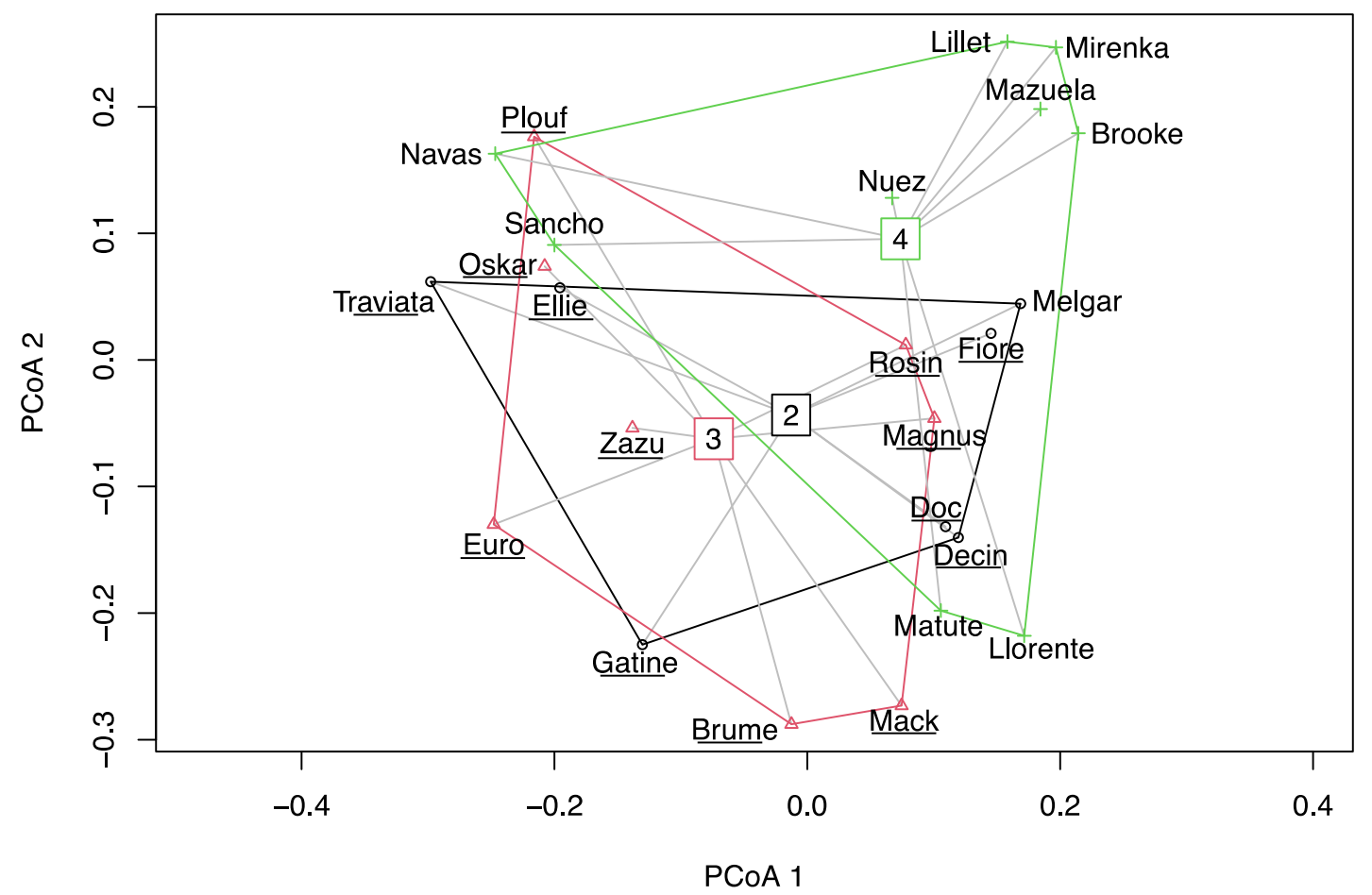

Figure 4. Differences in E-mink microbial community composition visualized by principal 620 coordinates analysis of unweighted UniFrac distances based on rarefied OTU counts. 621 Individuals are labelled according to their name their population or origin (animal names 622 from the eastern population are underlined, not underlined for western). Clusters 623 correspond to number of MHC-II motifs for each individual where the value is indicated 624 at the centroid. 


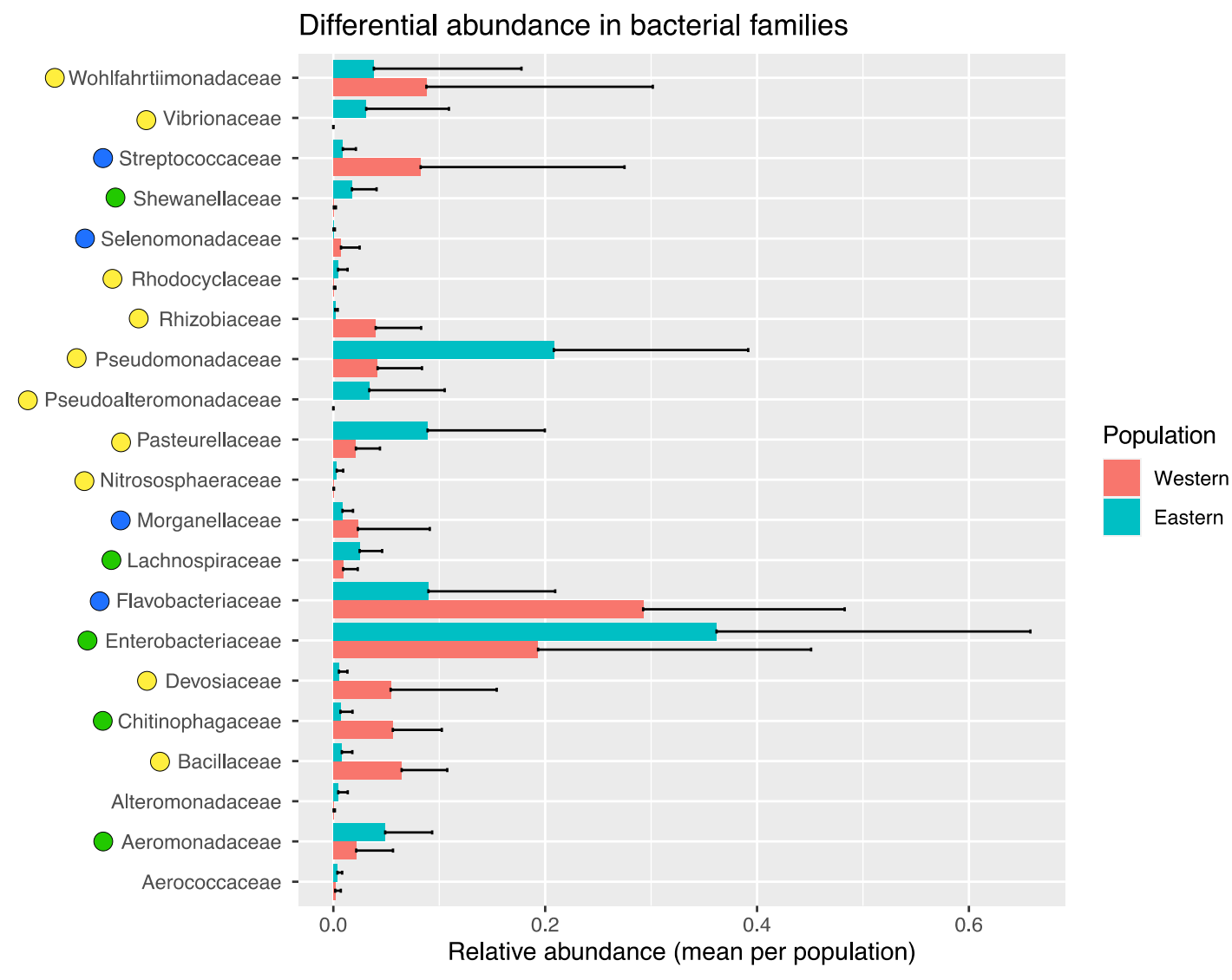

625

626

627

628

629

630

631

632

633

634

635

636

637

638

639

640

641

642

643

644

645

646

Figure 5. Mean relative abundance of each family that experienced significant differential abundance between the two mink populations (orange: Western; blue: Eastern, mean relative abundance per population + standard error). Colored circles next to the family name correspond to which MHC motifs absence/presence the variation in abundance was significant for (yellow: MHCII, blue: MHCI, green: both MHCI and MHCII).

\section{References}

[dataset] Van Leeuwen, P., Schulte-Hostedde, A., Fournier-Chambrillon, C., Aranda, M.C., Berthomieu, L., Fournier, P., Michaux, J.; 2021; Pooled 16S rRNA sequences SUB9956843; GenBank NCBI; DOI to be determined

[dataset] Van Leeuwen, P., Schulte-Hostedde, A., Fournier-Chambrillon, C., Aranda, M.C., Berthomieu, L., Fournier, P., Michaux, J.; 2021; ASV table, taxonomy table and mapping file have been uploaded, MHC-1, MHC-2 and microsatellite data; Dryad; DOI to be determined

Aguilar, A., Roemer, G., Debenham, S., Binns, M., Garcelon, D., \& Wayne, R. K. (2004). High MHC diversity maintained by balancing selection in an otherwise genetically monomorphic mammal. Proceedings of the National Academy of Sciences of the United States of America, 101(10), 3490-3494. doi: 10.1073/pnas.0306582101 
647

649

650

651

652

653

654

655

656

657

658

659

660

661

662

663

664

665

666

667

668

669

670

671

672

673

674

675

676

677

678

679

680

681

Andrews, S. (2010). FastQC: A quality control tool for high throughput sequence data. Available online at: http://www.bioinformatics.babraham.ac.uk/projects/fastqc

Becker, L., Nieberg, C., Jahreis, K., \& Peters, E. (2009). MHC class II variation in the endangered European mink Mustela lutreola (L . 1761) — consequences for species conservation. Immunogenetics, 61, 281-288. doi: 10.1007/s00251-009-0362-2

Bjorkman, P. J., \& Parham, P. (1990). Structure, function, and diversity of class I major histocompatibility complex molecules. Annual Review of Biochemistry (Vol. 59). doi: 10.1146/annurev.bi.59.070190.001345

Bokulich, N. A., Kaehler, B. D., Rideout, J. R., Dillon, M., Bolyen, E., Knight, R., ... Gregory Caporaso, J. (2018). Optimizing taxonomic classification of marker-gene amplicon sequences with QIIME 2's q2-feature-classifier plugin. Microbiome, 6(1), 1-17. doi: 10.1186/s40168-018-0470-Z

Bolnick, D. I., Snowberg, L. K., Caporaso, J. G., Lauber, C., Knight, R., \& Stutz, W. E. (2014). Major Histocompatibility Complex class IIb polymorphism influences gut microbiota composition and diversity. Molecular Ecology, 23(19), 4831-4845. doi: 10.1111/mec.12846

Bouman, J. (1977). The future of Przewalski horses (Equus przewalskii) in captivity. Int $\mathrm{Zoo} \mathrm{Yb} 17,62-68$.

Bolyen, E., Rideout. J. R., Dillon, M. R., Bokulich, N. A., Abnet, C., Al-Ghalith, G. A., Alexander, H., ... Caporaso, J. G. (2018). QIIME 2: Reproducible, interactive, scalable, and extensible microbiome data science. PeerJ Preprints6:e27295v2. doi: 10.7287 /peerj.preprints.27295v2

Bowling, A. T., Zimmermann, W., Ryder, O., Penado, C., Peto, S., Chemnick, L., ... Zharkikh, T. (2003). Genetic variation in Przewalski's horses, with special focus on the last wild caught mare, 231 Orlitza III. Cytogenetic and Genome Research, 102(1-4), 226-234. doi: 10.1159/000075754

Butchart, S. H. M., Walpole, M., Collen, B., Van Strien, A., Scharlemann, J. P. W., Almond, R. E. A., ... Watson, R. (2010). Global biodiversity: Indicators of recent declines. Science, 328(5982), 1164-1168. doi: 10.1126/science.1187512

Cabria, M. T., González, E. G., Gómez-Moliner, B. J., \& Zardoya, R. (2007). Microsatellite markers for the endangered European mink (Mustela lutreola) and closely related mustelids. Molecular Ecology Notes, 7(6), 1185-1188. doi: 10.1111/j.1471-8286.2007.01825.x

Cabria, M. T., Gonzalez, E. G., Gomez-Moliner, B. J., Michaux, J. R., Skumatov, D., Kranz, A., ... Zardoya, R. (2015). Patterns of genetic variation in the endangered 
European mink (Mustela lutreola L., 1761). BMC Evolutionary Biology, 15(1), 141. doi: 10.1186/s12862-015-0427-9

Callahan, B. J., Mcmurdie, P. J., Rosen, M. J., Han, A. W., Johnson, A. J. A., \& Holmes, S. P. (2016). DADA2: High-resolution sample inference from Illumina amplicon data. Nature Methods, 13(7), 581-588. doi: 10.1038/nmeth.3869

Callahan, B. J., Mcmurdie, P. J., \& Holmes, S. P. (2017). Exact sequence variants should replace operational taxonomic units in marker-gene data analysis. ISME Journal, 11(12), 2639-2643. doi: 10.1038/ismej.2017.119

Carbonell, R. (2015). Managing Spanish European mink populations: Moving from a precautionary approach towards knowledge-based management. Journal for Nature Conservation, 25, 58-61. doi: 10.1016/j.jnc.2015.03.004

Cardona, C., Lax, S., Larsen, P., Stephens, B., Hampton-Marcell, J., Edwardson, C. F., ... Gilbert, J. A. (2018). Environmental sources of bacteria differentially influence hostassociated microbial dynamics. Msystems, 3(3), e00052-e118. doi:10.1128/mSyst ems.00052-18

Ceballos, G., Ehrlich, P. R., Barnosky, A. D., García, A., Pringle, R. M., \& Palmer, T. M. (2015). Accelerated modern human-induced species losses: Entering the sixth mass extinction. Science Advances, 1(5), 9-13. doi: 10.1126/sciadv.1400253

Edwards, S. V., \& Hedrick, P. W. (1998). Evolution and ecology of MHC molecules: From genomics to sexual selection. TREE, 13(8), 443-453. doi: 10.1159/000103795

Evanno, G., Regnaut, S., Goudet, J. (2005). Detecting the number of clusters of individuals using the software Structure: A simulation study. Molecular Ecology, 14, 2611-2620.

Fleming, M. A., Ostrander, E. A., \& Cook, J. A. (1999). Microsatellite markers for American mink (Mustela vison) and ermine (Mustela erminea). Molecular Ecology, 8(8), 1351-1352. doi: 10.1046/j.1365-294X.1999.00701.x

Foster, K. R., Schluter, J., Coyte, K. Z., \& Rakoff-Nahoum, S. (2017). The evolution of the host microbiome as an ecosystem on a leash. Nature, 548(7665), 43-51. doi: 10.1038 /nature23292

Fournier-Chambrillon, C., Aasted, B., Perrot, A., Pontier, D., Sauvage, F., Artois, M., ... Fournier, P. (2004). Antibodies to Aleutian mink disease parvovirus in free-ranging European mink (Mustela lutreola) and other small carnivores from Southwestern France. Journal of Wildlife Diseases, 40(3), 394-402. doi: 10.7589/0090-355840.3.394 
716

717

718

719

720

721

722

723

724

725

726

727

728

729

730

731

732

733

734

735

736

737

738

739

740

741

742

743

744

745

746

747

748

749

Fournier-Chambrillon, C., Ceña, J. C., Urra Maya, F., Van De Bildt, M., Ferreras, M. C., Giralda-Carrera, G., Kuiken, T., Buisson, L., Palomares, F., \& Fournier, P. (2022). A 9-year demographic and health survey of an European mink population in Navarre (Spain): role of the canine distemper virus. In: Small Carnivores in Space and Time: Evolution, Ecology, Behaviour and Conservation, E. Do Linh San, J. J. Sato, J. L. Belant and M. J. Somers (eds). Wiley, Chichester, United Kingdom, pp. In print.

Frankham, R. (2005). Genetics and extinction. Biol. Conserv. 126, 131-140.

Frankham, R. (2008). Genetic adaptation to captivity in species conservation programs. Molecular Ecology, 17(1), 325-333. doi: 10.1111/j.1365-294X.2007.03399.x

Gould, A. L., Zhang, V., Lamberti, L., Jones, E. W., Obadia, B., Korasidis, N., ... Ludington, W. B. (2018). Microbiome interactions shape host fitness. Proceedings of the National Academy of Sciences of the United States of America, 115(51), E11951-E11960. doi: 10.1073/pnas.1809349115

Grosser, S., Sauer, J., Paijmans, A. J., Caspers, B. A., Forcada, J., Wolf, J. B. W., \& Hoffman, J. I. (2019). Fur seal microbiota are shaped by the social and physical environment, show mother-offspring similarities and are associated with host genetic quality. Molecular Ecology, 28(9), 2406-2422. doi: 10.1111/mec.15070

Hapke, A. (2004). Population genetics and differentiation of species of the genus of mouse lemurs, Microcebus (E. Geoffroy St. Hilaire, 1828) and dwarf lemurs, Cheirogaleus (E. Geoffrey St. Hilaire, 1812) in southeastern Madagascar. In PhD Thesis University Hamburg, Germany.

Hedrick, P. W. (1999). Balancing selection and MHC. Genetica, 104(3), 207-214. doi: 10.1023/A:1026494212540

Hedrick, P. W. (2001). Conservation genetics: Where are we now? Trends in Ecology \& Evolution, 16(11), 629-636. doi: 10.1016/S0169-5347(01)02282-0

Hughes, A. L. (1991). MHC polymorphism and the design of captive breeding programs. Conservation Biology, 5(2), 249-251. doi: 10.1111/j.1523-1739.1991.tb00130.x

Jakobsson, M., Rosenberg, N. A. (2007). Clumpp: a cluster matching and permutation program for dealing with label switching and multimodality in analysis of population structure. Bioinformatics, 23, 1801-1806.

Jarvi, S. I., Tarr, C. L., Mcintosh, C. E., Atkinson, C. T., \& Fleischer, R. C. (2004). Natural selection of the major histocompatibility complex (MHC) in Hawaiian honeycreepers (Drepanidinae). Molecular Ecology, 13(8), 2157-2168. doi: 10.1111/j.1365-294X.2004.02228.x 
750

751

752

753

754

755

756

757

758

759

760

761

762

763

764

765

766

767

768

769

770

771

772

773

774

775

776

777

778

779

780

781

782

783

784

Khan, M. A. W., Id, W. Z. S., Mohammed, A. D., Round, J. L., Lee, J., \& Id, K. (2019). Does MHC heterozygosity influence microbiota form and function? PLoS ONE, 14(5), e0215946.

Kiik, K., Maran, T., Nagl, A., Ashford, K., \& Tammaru, T. (2013). The causes of the low breeding success of European mink (Mustela lutreola) in captivity. Zoo Biology, 32(4), 387-393. doi: 10.1002/zoo.21062

Kopelman, N. M., Mayzel, J., Jakobsson, M., Rosenberg, N. A., Mayrose, I. (2015). CLUMPAK: a program for identifying clustering modes and packaging population structure inferences across K. Molecular Ecology Resources, 15(5), 1179-1191.

Koskella, B., Hall, L. J., \& Metcalf, C. J. E. (2017). The microbiome beyond the horizon of ecological and evolutionary theory. Nature Ecology \& Evolution, 1(November). doi: 10.1038/s41559-017-0340-2

Kubinak, J. L., Ruff, J. S., Hyzer, C. W., Slev, P. R., \& Potts, W. K. (2012). Experimental viral evolution to specific host MHC genotypes reveals fitness and virulence tradeoffs in alternative MHC types. Proceedings of the National Academy of Sciences of the United States of America, 109(9), 3422-3427. doi: 10.1073/pnas.1112633109

Larson, S. (2012). Loss of genetic diversity in wild populations, analysis of genetic variation in animals, Prof. Mahmut Caliskan (Ed.), ISBN: 978-953-51-0093-5, InTech

Leclaire, S., Strandh, M., Dell'Ariccia, G., Gabirot, M., Westerdahl, H., \& Bonadonna, F. (2018). Plumage microbiota covaries with MHC in blue petrels. Molecular Ecology, 0-2. doi: 10.1111/mec.14993

Love, M. I., Huber, W., \& Anders, S. (2014). Moderated estimation of fold change and dispersion for RNA-seq data with DESeq2. Genome Biology, 15(12), 550. doi: 10.1186/s13059-014-0550-8

Lozupone, C., \& Knight, R. (2005). UniFrac: A new phylogenetic method for comparing microbial communities. Applied and Environmental Microbiology, 71(12), 82288235. doi: 10.1128/AEM.71.12.8228

Mallinson, J. J. C. (1995). Conservation breeding programs: an important ingredient for species survival. Biodiversity and Conservation, 4(6), 617-635. doi: 10.1007/BF00222518

Mañas, S., Gómez, A., Asensio, V., Palazón, S., Podra, M., Alarcia, O. E., ... Casal, J. (2016). Prevalence of antibody to Aleutian mink disease virus in European mink (Mustela lutreola) and American mink (Neovison vison) in Spain. Journal of Wildlife Diseases, 52(1), 22-32. doi: 10.7589/2015-04-082 
Maran, T., Skumatov, D., Gomez, A., Põdra, M., Abramov, A.V. \& Dinets, V., (2016). Mustela lutreola. The IUCN Red List of Threatened Species 2016: e.T14018A45199861. http://dx.doi.org/10.2305/IUCN.UK.20161.RLTS.T14018A45199861.en.

Marsden, C. D., Verberkmoes, H., Thomas, R., Wayne, R. K., \& Mable, B. K. (2013). Pedigrees, MHC and microsatellites: An integrated approach for genetic management of captive African wild dogs (Lycaon pictus). Conservation Genetics, 14(1), 171-183. doi: 10.1007/s10592-012-0440-0

Marshall, T. C., Sunnucks, P., Spalton, J. A., Greth, A., \& Pemberton, J. M. (1999). Use of genetic data for conservation management: The case of the Arabian oryx. Animal Conservation, 2(4), 269-278. doi: 10.1111/j.1469-1795.1999.tb00073.x

McKenzie, V. J., Song, S. J., Delsuc, F., Prest, T. L., Oliverio, A. M., Korpita, T. M., ... Knight, R. (2017). The effects of captivity on the mammalian gut microbiome. Integrative and Comparative Biology, 1-15. doi: 10.1093/icb/icx090

Michaux, J. R., Hardy, O. J., Justy, F., Fournier, P., Kranz, A., Cabria, M., ... Libois, R. (2005). Conservation genetics and population history of the threatened European mink Mustela lutreola, with an emphasis on the west European population. Molecular Ecology, 14(8), 2373-2388. doi: 10.1111/j.1365-294X.2005.02597.x

Mueller, E. A., Wisnoski, N. I., Peralta, A. L., \& Lennon, J. T. (2019). Microbial rescue effects: How microbiomes can save hosts from extinction. Functional Ecology, (November 2019), 1-10. doi: 10.1111/1365-2435.13493

Origgi, F. C., Plattet, P., Sattler, U., Robert, N., Casaubon, J., Mavrot, F., ... RyserDegiorgis, M. P. (2012). Emergence of canine distemper virus strains with modified molecular signature and enhanced neuronal tropism leading to high mortality in wild carnivores. Veterinary Pathology, 49(6), 913-929. doi: 10.1177/0300985812436743

Ost, K. S., \& Round, J. L. (2018). Communication between the microbiota and mammalian immunity. Annual Review of Microbiology, 72(June), 399-422. doi: 10.1146/annurev-micro-090817-062307

Parmar, D. R., Mitra, S., Bhadouriya, S., Rao, T., Kunteepuram, V., \& Gaur, A. (2017). Characterization of major histocompatibility complex class I, and class II DRB loci of captive and wild Indian leopards (Panthera pardus fusca). Genetica, 145(6), 541558. doi: 10.1007/s10709-017-9979-5

Peakall, R. and Smouse P.E. (2006) GENALEX 6: Genetic analysis in Excel. Population genetic software for teaching and research. Molecular Ecology Notes. 6, 288-295. 
819

820

821

822

823

824

825

826

827

828

829

830

831

832

833

834

835

836

837

838

839

840

841

842

843

844

845

846

847

848

849

850

851

852

853

Pelletier, F., Réale, D., Watters, J., Boakes, E. H., \& Garant, D. (2009). Value of captive populations for quantitative genetics research. Trends in Ecology and Evolution, 24(5), 263-270. doi: 10.1016/j.tree.2008.11.013

Penn, D. J., \& Potts, W. K. (1999). The evolution of mating preferences and major histocompatibility complex genes. The American Naturalist, 153(2), 145-164. doi: $10.1086 / 303166$

Penn, D. J. (2002). The scent of genetic compatibility: Sexual selection and the major histocompatibility complex. Ethology, 108, 1-21.

Philippa, J., Fournier-Chambrillon, C., Fournier, P., Schaftenaar, W., van de Bildt, M., van Herweijnen, R., ... Osterhaus, A. (2008). Serologic survey for selected viral pathogens in free-ranging endangered European mink (Mustela lutreola) and other mustelids from South-Western France. Journal of Wildlife Diseases, 44(4), 791801. doi: 10.7589/0090-3558-44.4.791

Pigneur, L. M., Caublot, G., Fournier-Chambrillon, C., Fournier, P., Giralda-Carrera, G., Grémillet, X., ... Michaux, J. R. (2019). Current genetic admixture between relictual populations might enhance the recovery of an elusive carnivore. Conservation Genetics, 20(5), 1133-1148. doi: 10.1007/s10592-019-01199-9

Pritchard, J. K., Stephens, M., Donnelly, P. (2000). Inference of population structure using multilocus genotype data. Genetics, 155, 945-959.

Pruesse, E., Quast, C., Knittel, K., Fuchs, B. M., Ludwig, W. G., Peplies, J., Glöckner, F. O. (2007). SILVA: A comprehensive online resource for quality checked and aligned ribosomal RNA sequence data compatible with ARB. Nucleic Acids Research, 35,7188-7196. doi: 10.1093/nar/gkm864

R Development Core Team (2008) R: A language and environment for statistical computing. R Foundation for Statistical Computing, Vienna.

Ralls, K., Brugger, K., \& Ballou, J. (1975). Inbreeding and juvenile mortality in small populations of ungulates: A detailed analysis. Biological Conservation, 206(November), 1101-. doi: 10.1016/0006-3207(82)90014-3

Reche, P. A., \& Reinherz, E. L. (2003). Sequence variability analysis of human class I and class II MHC molecules: Functional and structural correlates of amino acid polymorphisms. Journal of Molecular Biology, 331(3), 623-641. doi: 10.1016/S0022-2836(03)00750-2

Reed, D. H., \& Frankham, R. (2001). How closely correlated are molecular and quantitative measures of genetic variation? A meta-analysis. Evolution, 55(6), 10951103. doi: 10.1111/j.0014-3820.2001.tb00629.x 
854 Rico, Y., Ethier, D. M., Davy, C. M., Sayers, J., Weir, R. D., Swanson, B. J., ... Kyle, C. 855 J. (2016). Spatial patterns of immunogenetic and neutral variation underscore the conservation value of small, isolated American badger populations. Evolutionary Applications, 9(10), 1271-1284. doi: 10.1111/eva.12410

Schad, J., Sommer, S., \& Ganzhorn, J. U. (2004). MHC variability of a small lemur in the littoral forest fragments of southeastern Madagascar. Conservation Genetics, 5, 299309.

Schulte-Hostedde, A. I., \& Mastromonaco, G. F. (2015). Integrating evolution in the management of captive zoo populations. Evolutionary Applications, 8(5), 413-422. doi: 10.1111/eva.12258

Sebastian, A., Herdegen, M., Migalska, M., \& Radwan, J. (2016). Amplisas: A web server for multilocus genotyping using next-generation amplicon sequencing data. Molecular Ecology Resources, 16(2), 498-510. doi: 10.1111/1755-0998.12453

Selkoe, K. A., \& Toonen, R. J. (2006). Microsatellites for ecologists: a practical guide to using and evaluating microsatellite markers. Ecology Letters, 9(5), 615-629. doi: 10.1111/j.1461-0248.2006.00889.x

Signer, E. N., Schmidt, C. R., Jeffreys, A. J. (1994). DNA variability and parentage testing in captive Waldrapp ibises. Molecular Ecology, 3, 291-300.

Sin, Y. W., Dugdale, H. L., Newman, C., Macdonald, D. W., \& Burke, T. (2012). Evolution of MHC class I genes in the European badger (Meles meles). Ecology and Evolution, 2(7), 1644-1662. doi: 10.1002/ece3.285

Sommer, S. (2005). The importance of immune gene variability (MHC) in evolutionary ecology and conservation. Frontiers in Zoology, 2, 1-18. doi: 10.1186/1742-9994-216

Spor, A., Koren, O., \& Ley, R. (2011). Unravelling the effects of the environment and host genotype on the gut microbiome. Nature Reviews Microbiology, 9(4), 279-290. doi: $10.1038 /$ nrmicro 2540 being inbred. Current Biology, 29(16), R796-R798. doi: 10.1016/j.cub.2019.07.023

Strandh, M., Westerdahl, H., Pontarp, M., Canbäck, B., Dubois, M. P., Miquel, C., ... Bonadonna, F. (2012). Major histocompatibility complex class II compatibility, but not class I, predicts mate choice in a bird with highly developed olfaction. Proceedings of the Royal Society B: Biological Sciences, 279(1746), 4457-4463. doi: $10.1098 / \mathrm{rspb} .2012 .1562$ 
888

889

890

891

892

893

894

895

896

897

898

899

900

901

902

903

904

905

906

907

908

909

910

911

912

913

914

915

916

917

918

919

Trevelline, B. K., Fontaine, S. S., Hartup, B. K., \& Kohl, K. D. (2019). Conservation biology needs a microbial renaissance: A call for the consideration of hostassociated microbiota in wildlife management practices. Proceedings of the Royal Society Biological Sciences, 286, 1-9. doi: 10.1098/rspb.2018.2448

Ujvari, B., \& Belov, K. (2011). Major histocompatibility complex (MHC) markers in conservation biology. International Journal of Molecular Sciences, 12(8), 51685186. doi: 10.3390/ijms 12085168

van Leeuwen, P., Mykytczuk, N., Mastromonaco, G. F., \& Schulte-Hostedde, A. I. (2020). Effects of captivity, diet, and relocation on the gut bacterial communities of white-footed mice. Ecology and Evolution, 10(11), 4677-4690. doi: 10.1002/ece3.6221

Wasimuddin, Menke, S., Melzheimer, J., Thalwitzer, S., Heinrich, S., Wachter, B., \& Sommer, S. (2017). Gut microbiomes of free-ranging and captive Namibian cheetahs: Diversity, putative functions and occurrence of potential pathogens. Molecular Ecology, 26(20), 5515-5527. doi: 10.1111/mec.14278

West, A. G., Waite, D. W., Deines, P., Bourne, D. G., Digby, A., Mckenzie, V. J., \& Taylor, M. W. (2019). The microbiome in threatened species conservation. Biological Conservation, 229(November 2018), 85-98. doi: 10.1016/j.biocon.2018.11.016

Williams, S. E., \& Hoffman, E. A. (2009). Minimizing genetic adaptation in captive breeding programs: A review. Biological Conservation, 142(11), 2388-2400. doi: 10.1016/j.biocon.2009.05.034

Willoughby, J. R., \& Christie, M. R. (2019). Long-term demographic and genetic effects of releasing captive-born individuals into the wild. Conservation Biology, 33(2), 377-388. doi: 10.1111/cobi.13217

Witzenberger, K. A., \& Hochkirch, A. (2011). Ex situ conservation genetics: A review of molecular studies on the genetic consequences of captive breeding programs for endangered animal species. Biodiversity and Conservation, 20(9), 1843-1861. doi: 10.1007/s10531-011-0074-4

Woodworth, L. M., Montgomery, M. E., Briscoe, D. A., \& Frankham, R. (2002). Rapid genetic deterioration in captive populations: Causes and conservation implications. Conservation Genetics, 3(3), 277-288. doi: 10.1023/A:1019954801089 


\section{Supplementary Files}

This is a list of supplementary files associated with this preprint. Click to download.

- minksmicrobesmhcsuppjuly.docx 